\title{
MODELADO CINEMÁTICO Y SIMULACIÓN REALISTA DEL MANIPULADOR MÓVIL DE BAJO COSTE TURTLEBOT2 + WIDOW-X EN ROS
}

\author{
Marina Aguilar, Ana Cruz-Martín y Juan-Antonio Fernández-Madrigal \\ Departamento de Ingeniería de Sistemas y Automática \\ Universidad de Málaga \\ marinaaguilar@uma.es, anacm@uma.es, jafernandez@uma.es
}

\begin{abstract}
Resumen
El manipulador móvil compuesto de la plataforma Turtlebot2 y el brazo Widow-X supone una solución de bajo coste muy interesante para laboratorios docentes y de investigación. Lamentablemente, el conjunto no dispone de un simulador completo en el popular framework de programación ROS; tampoco el fabricante ofrece el modelado cinemático del brazo. En este artículo se describe el trabajo realizado sobre ambas cuestiones para nuestro robot CRUMB, y cómo se han implementado en Gazebo, un simulador realista para ROS donde también se ha añadido la posibilidad de incluir ruido sensorial simple, algo que la mayoría de los simuladores no ofrecen. Se incluyen también algunos experimentos de manipulación y navegación que demuestran las posibilidades de nuestro trabajo.
\end{abstract}

Palabras clave: manipulador móvil, simulación, modelado cinemático, Turtlebot2, Widow-X, ROS, Gazebo.

\section{INTRODUCCIÓN}

Como parte del proyecto puente de investigación denominado CRUMB [1] (Cognitive-RoboticssUpporting Mobile Base), financiado a través del Plan Propio de Investigación de la Universidad de Málaga, se adquirió un manipulador móvil de bajo coste sobre el que implementar desarrollos de Robótica Cognitiva. Este robot está formado por una base móvil Turtlebot2 [2] y un brazo manipulador Widow-X [3], mostrados en la figura 1.

Para facilitar que varios miembros del equipo investigador pudieran trabajar de manera simultánea con esta plataforma robótica, se decidió crear un simulador realista compatible con el robot real, de manera que los algoritmos desarrollados en el primero pudieran utilizarse en el robot $C R U M B$ con mínimos cambios, y que se incorporaran efectos suficientemente realistas en cinemática, dinámica y ruido de los sensores. Como

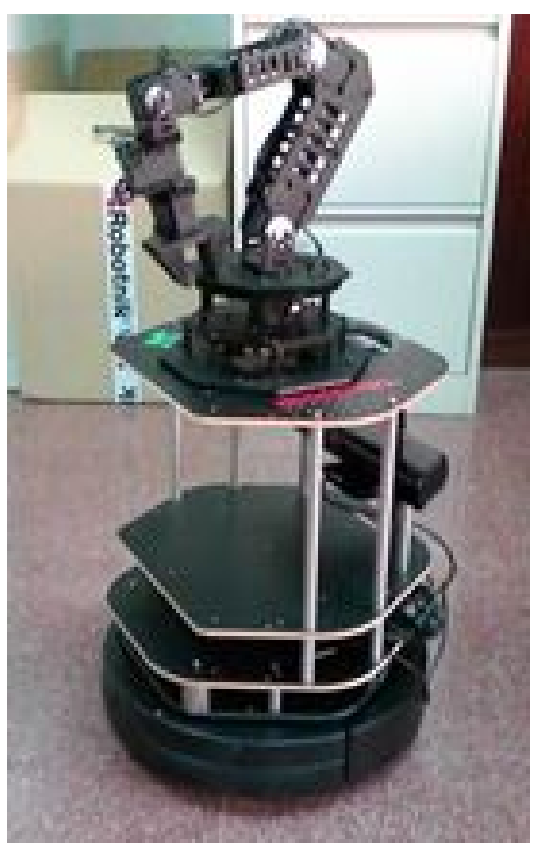

Figura 1: Manipulador móvil CRUMB.

el robot debía ser capaz de realizar tareas de manipulación, en primer lugar era necesario obtener el modelo cinemático del brazo manipulador Widow$X$, no disponible por parte del fabricante, y resolver los problemas directo e inverso. Además, el simulador del robot completo debía estar integrado en $R O S$ [4], dada su popularidad como framework de programación en la comunidad Robótica y por tanto, su idoneidad para compartir desarrollos de diversos investigadores.

En este artículo se presenta el trabajo realizado bajo los mencionados objetivos, concretamente el modelado cinemático del brazo y la simulación realista del manipulador móvil en Gazebo [5] (que se encarga de la dinámica realista), así como la implementación de un interfaz de programación prácticamente idéntico al del robot real (es decir, los topics de ROS necesarios) y la inclusión de ruido simple en los sensores. 
El texto se organiza de la siguiente forma: en la sección 2 se describe el cálculo del modelado cinemático directo e inverso del brazo manipulador Widow- $X$; en la sección 3 se detalla cómo se ha implementado el simulador para $C R U M B$ en $R O S$ y Gazebo, incluyendo el modelado geométrico y de los parámetros físicos y de control que hemos realizado; en la sección 4 se muestran los experimentos realizados con el simulador completo; finalmente, en la sección 5 se resumen las conclusiones y las líneas de trabajo futuras.

\section{PROBLEMAS CINEMÁTICO DIRECTO E INVERSO EN EL BRAZO WIDOW-X}

Para realizar tareas de manipulación (p.ej., pick and place) con el brazo Widow-X es necesario al menos su modelado cinemático. Este brazo cuenta con 5 g.d.l y una pinza paralela como efector final.

El modelo cinemático se basará en el producto de las matrices de paso correspondientes a cada una de las articulaciones. Para calcular dichas matrices de paso se necesitan los parámetros de DenavitHartenberg [6], que son (ver figura 2):

- $a_{i}$ : distancia desde el eje $Z_{i}$ hasta el eje $Z_{i+1}$ medida a lo largo de $X_{i}$.

- $\alpha_{i}$ : ángulo que forman los ejes $Z_{i}$ y $Z_{i+1}$ medido sobre $X_{i}$.

- $d_{i}$ : distancia desde el eje $X_{i-1}$ hasta el eje $X_{i}$ medida a lo largo de $Z_{i}$.

- $\theta_{i}$ : ángulo que forman los ejes $X_{i-1}$ y $X_{i}$ medido sobre $Z_{i}$.

En el caso del Widow-X, estos parámetros son los mostrados en el cuadro 1. Con ellos se obtienen las matrices de paso $1,2,3,4$ y 5 .

Cuadro 1: Parámetros D-H del manipulador Widow-X.

\begin{tabular}{|c|c|c|c|c|}
\hline Articulación & $a_{i-1}$ & $\alpha_{i-1}$ & $d_{i}$ & $\theta_{i}$ \\
\hline 1 & 0 & 0 & 0 & $\theta_{1}$ \\
2 & 0 & $-\pi / 2$ & 0 & $\theta_{2}-\beta$ \\
3 & $\mathrm{~d} 2$ & $\pi$ & 0 & $\theta_{3}-\gamma$ \\
4 & $\mathrm{~L} 3$ & 0 & 0 & $\theta_{4}+\pi / 2$ \\
5 & 0 & $\pi / 2$ & 0 & $\theta_{5}$ \\
\hline
\end{tabular}

$$
T_{1}^{0}=\left[\begin{array}{cccc}
\cos \theta_{1} & -\sin \theta_{1} & 0 & 0 \\
\sin \theta_{1} & \cos \theta_{1} & 0 & 0 \\
0 & 0 & 1 & 0 \\
0 & 0 & 0 & 1
\end{array}\right]
$$

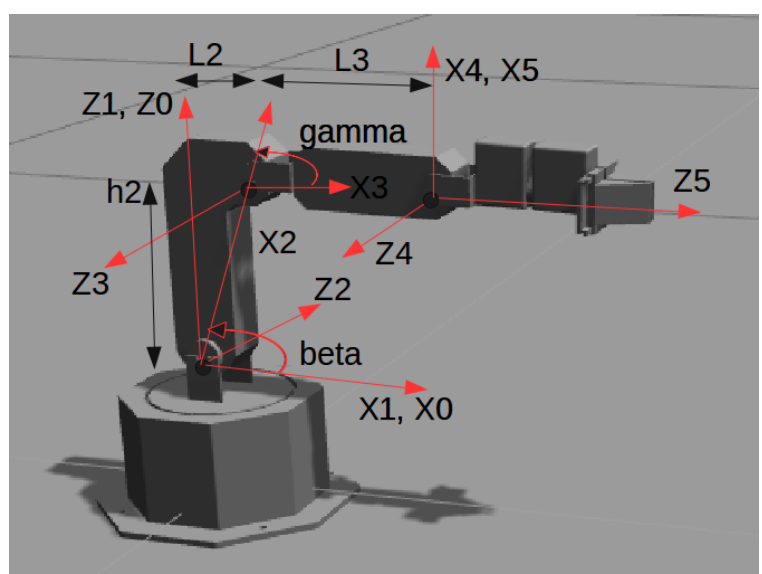

Figura 2: Ejes y dimensiones en el Widow-X para el cálculo de los parámetros Denavit-Hartenberg.

$T_{2}^{1}=\left[\begin{array}{cccc}\cos \left(\theta_{2}-\beta\right) & \sin \left(\theta_{2}-\beta\right) & 0 & 0 \\ 0 & 0 & 1 & 0 \\ \sin \left(\theta_{2}-\beta\right) & -\cos \left(\theta_{2}-\beta\right) & 0 & 0 \\ 0 & 0 & 0 & 1\end{array}\right]$

$T_{3}^{2}=\left[\begin{array}{cccc}\cos \left(\theta_{3}-\beta\right) & \sin \left(\theta_{3}-\beta\right) & 0 & d_{2} \\ \sin \left(\theta_{3}-\beta\right) & -\cos \left(\theta_{3}-\beta\right) & 0 & 0 \\ 0 & 0 & -1 & 0 \\ 0 & 0 & 0 & 1\end{array}\right]$

$T_{4}^{3}=\left[\begin{array}{cccc}\cos \left(\theta_{4}+\pi / 2\right) & -\sin \left(\theta_{4}+\pi / 2\right) & 0 & L_{3} \\ \sin \left(\theta_{4}+\pi / 2\right) & \cos \left(\theta_{4}+\pi / 2\right) & 0 & 0 \\ 0 & 0 & 1 & 0 \\ 0 & 0 & 0 & 1\end{array}\right]$

$$
T_{5}^{4}=\left[\begin{array}{cccc}
\cos \theta_{5} & -\sin \theta_{5} & 0 & 0 \\
0 & 0 & -1 & 0 \\
\sin \theta_{5} & \cos \theta_{5} & 0 & 0 \\
0 & 0 & 0 & 1
\end{array}\right]
$$

El modelo cinemático será el indicado en 6. El punto obtenido con el mismo será el nombrado como $O_{m}$ en la figura 3 , que corresponde a la muñeca.

$$
T_{5}^{0}=T_{1}^{0} T_{2}^{1} T_{3}^{2} T_{4}^{3} T_{5}^{4}
$$

El problema cinemático directo consiste básicamente en la realización del producto anterior con una postura articular dada. El inverso necesita la resolución de las mismas ecuaciones cuando tal postura es desconocida. En nuestro caso se ha obtenido con un procedimiento algebraico mediante desacoplo de la muñeca respecto del brazo: en primer lugar se obtienen las variables articulares $\theta_{1}$, 


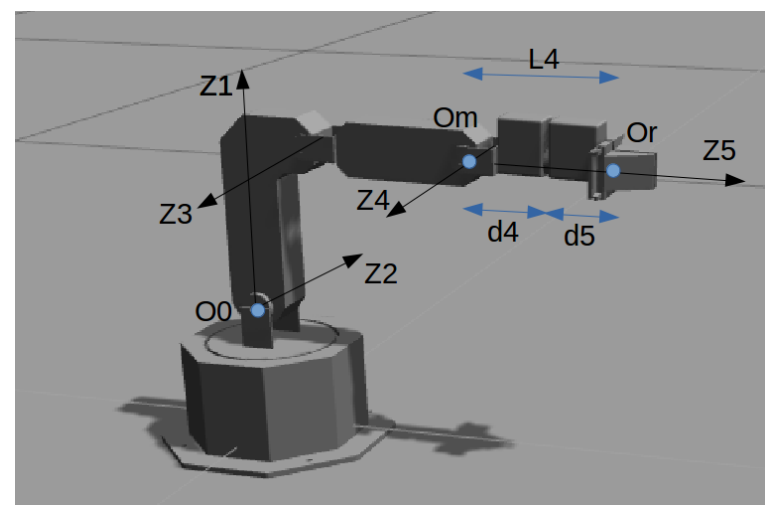

Figura 3: Posición de la muñeca y efector final.

$\theta_{2}$ y $\theta_{3}$ en función de un punto genérico, y a continuación $\theta_{4}$ y $\theta_{5}$ para una orientación genérica de la muñeca.

Para ello, partiendo de la matriz $T_{5}^{0}$, se modifica la última columna para introducir el punto genérico de la muñeca $\left(p_{x}, p_{y}, p_{z}\right)$, obteniéndose la matriz $T p_{5}^{0}$ de la ecuación 7 , en la que los elementos $r_{i j}$ son los elementos $i j$ del modelo cinemático.

$$
T p_{5}^{0}=\left[\begin{array}{cccc}
r_{11} & r_{12} & r_{13} & p_{x} \\
r_{21} & r_{22} & r_{23} & p_{y} \\
r_{31} & r_{32} & r_{33} & p_{z} \\
0 & 0 & 0 & 1
\end{array}\right]
$$

La matriz $T p_{5}^{0}$ debe ser igual a $T_{5}^{0}$, con lo que se obtiene la ecuación 8d.

$$
\begin{aligned}
T p_{5}^{0} & =T_{1}^{0} T_{2}^{1} T_{3}^{2} T_{4}^{3} T_{5}^{4} \\
{\left[T_{1}^{0}\right]^{-1} T p_{5}^{0} } & =T_{2}^{1} T_{3}^{2} T_{4}^{3} T_{5}^{4} \\
T_{0}^{1} T p_{5}^{0} & =T_{2}^{1} T_{3}^{2} T_{4}^{3} T_{5}^{4} \\
T p_{5}^{1} & =T_{2}^{1} T_{3}^{2} T_{4}^{3} T_{5}^{4}
\end{aligned}
$$

Resolviendo el sistema de ecuaciones resultante se obtienen las variables articulares $\theta_{1}, \theta_{2}$ y $\theta_{3}$ (ecuaciones $9 \mathrm{a}, 9 \mathrm{~b}$ y $9 \mathrm{c}$, respectivamente).

$$
\begin{aligned}
\theta_{1}= & A \tan 2\left(p_{y}, p_{x}\right) \\
\theta_{2}= & \beta+A \tan 2\left(k_{1}, k_{2}\right)- \\
& A \tan 2\left(k, \pm \sqrt{k_{1}^{2}+k_{2}^{2}+k^{2}}\right) \\
\theta_{3}= & \theta_{2}-A \sin \left(\frac{-p_{z}-d_{2} \sin \beta}{L_{3}}\right)
\end{aligned}
$$

donde $k, k_{1}$ y $k_{2}$ son, respectivamente, 10a, 10b y $10 \mathrm{c}$.

$$
\begin{aligned}
k= & p_{z}^{2}+d_{2}^{2}-L_{3}^{2}+ \\
& \left(p_{x} \cos \theta_{1}+p_{y} \sin \theta_{1}\right)^{2} \\
k_{1}= & 2 p_{x} \cos \theta_{1} d_{2}+2 p_{y} \sin \theta_{1} d_{2} \\
k_{2}= & 2 p_{z} d_{2}
\end{aligned}
$$

Para obtener $\theta_{4}$ y $\theta_{5}$, se utiliza la orientación de la muñeca. Sea $R_{5}^{0}$ la submatriz de rotación de $T_{5}^{0}$ para una orientación genérica $[\hat{n}, \hat{o}, \hat{a}]$ (ecuación 11).

$$
R_{5}^{0}=[\hat{n}, \hat{o}, \hat{a}]=\left[\begin{array}{lll}
n_{x} & o_{x} & a_{x} \\
n_{y} & o_{y} & a_{y} \\
n_{z} & o_{z} & a_{z}
\end{array}\right]
$$

Se tiene la igualdad 12:

$$
R_{5}^{0}=R_{3}^{0} \cdot R_{5}^{3}
$$

donde $R_{3}^{0}$ es conocido ya que es el producto en la ecuación 13a de las submatrices de rotación mostradas, que dependen de $\theta_{1}, \theta_{2}$ y $\theta_{3}$, ya obtenidos previamente, y $R_{5}^{3}$ el producto de la ecuación $13 \mathrm{~b}$, cuyo resultado depende de $\theta_{4}$ y $\theta_{5}$.

$$
\begin{aligned}
& R_{3}^{0}=R_{1}^{0} \cdot R_{2}^{1} \cdot R_{3}^{2} \\
& R_{5}^{3}=R_{4}^{3} \cdot R_{5}^{4}
\end{aligned}
$$

$\mathrm{Al}$ resolver el sistema de ecuaciones resultante se obtienen las variables articulares de la muñeca, ecuaciones 14a y 14b.

$$
\begin{aligned}
& \theta_{4}=A \cos \left(-k_{3}\right)-\frac{\pi}{2} \\
& \theta_{5}=A \sin \left(n_{x} \sin \theta_{1}-n_{y} \cos \theta_{1}\right)
\end{aligned}
$$

donde $k_{3}$ es:

$$
\begin{aligned}
k_{3}= & a_{z} \cos \left(\theta_{2}-\theta_{3}\right)+ \\
& a_{x} \sin \left(\theta_{2}-\theta_{3}\right) \cos \theta_{1}+ \\
& a_{y} \sin \left(\theta_{2}-\theta_{3}\right) \sin \theta_{1}
\end{aligned}
$$

Finalmente, se obtiene la postura del efector final $O_{r}$ en función de la de la muñeca $O_{m}$, obtenida a su vez del modelo cinemático (figura 3 ), de donde se deduce la ecuación 16.

$$
\overrightarrow{P_{m}}=\overrightarrow{P_{r}}-L_{4} \cdot \hat{Z}_{5}
$$

\section{MODELADO DEL ROBOT PARA SU SIMULACIÓN}

En esta sección se describe el modelado geométrico, físico y de controladores necesario para la simulación en Gazebo del brazo manipulador Widow-X, así como el de la base móvil Turtlebot2 y del robot completo.

\subsection{BRAZO WIDOW-X}

El modelado del brazo Widow- $X$ se divide en modelado geométrico, modelado de parámetros físicos, modelado de los controladores y configuración de los plugins necesarios en Gazebo [7]. 


\subsubsection{Modelado geométrico}

La geometría del brazo se ha modelado partiendo de los ficheros CAD .stl facilitados por Robotnik Automation S.L.L. [8], distribuidores de este manipulador. Dicho modelado se describe en formato $X M L$, donde se diferencian dos elementos: eslabones y articulaciones.

En los eslabones se indican dos tipos de geometrías: la visible y la de detección de colisiones, donde se añade el fichero .stl junto con el origen de coordenadas y la orientación del eslabón. Para indicar la relación entre eslabones se utilizan las articulaciones. En este trabajo se utilizan uniones de revolución, prismáticas y fijas. En las dos primeras se indica el punto de contacto, el eje de giro, los límites del movimiento y cuáles son los eslabones fijo y móvil. También se puede indicar si una pieza imita el movimiento de otra, como en el caso de la pinza. Para las uniones fijas se indican las dos piezas que se unen, en este caso la base del brazo con la superficie superior de la base móvil.

También es necesario añadir propiedades de color y autocolisión en los ficheros de configuración, para que el modelo utilice la detección de colisiones para no atravesarse a sí mismo y para mejorar su aspecto visual.

\subsubsection{Modelado de parámetros físicos}

Para que el modelo sea lo más realista posible se han incluido en el mismo parámetros físicos como la inercia, el amortiguamiento y la fricción.

Para cada uno de los eslabones se ha calculado la masa, el centro de masas y el tensor de inercia respecto al centro de masas. El software utilizado ha sido MeshLab [9] para los centros de masa y $V$-REP [10] para la masa y el tensor de inercia.

Para comprobar que las inercias son correctas $G a-$ zebo dispone de un visor. En la figura 4 se observa, por ejemplo, cómo la geometría del brazo y la generada por la inercia son coincidentes.

El amortiguamiento y la fricción de los eslabones se han obtenido experimentalmente bajo la premisa de que el brazo no presente vibraciones. Se ha aumentado la resolución de los métodos numéricos durante la simulación para mejorar los resultados obtenidos.

Finalmente, para poder realizar tareas con la pinza hay que indicar cuáles son las partes móviles y fijas de la misma, el tiempo para agarrar y soltar el objeto y el rozamiento y el contacto. Todos estos parámetros se han obtenido de manera experimental persiguiendo que las piezas no se queden pegadas ni resbalen.

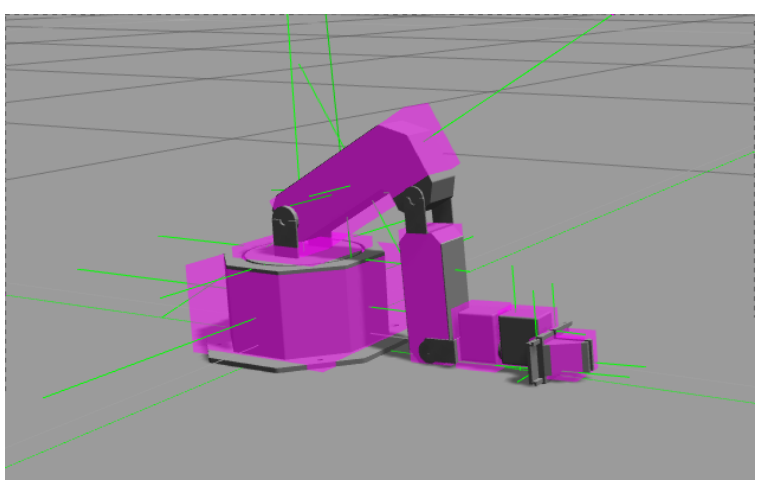

Figura 4: Widow-X en el visor de inercia de $G a$ zebo.

\subsubsection{Modelado de controladores}

Se ha elegido un control de esfuerzo/posición para la simulación de las articulaciones, ya que experimentalmente es el que mejores resultados ha ofrecido. El controlador PID se ha sintonizado de manera experimental.

También se han incluido el esfuerzo y la velocidad máxima de las articulaciones. Para estos datos se han utilizado los del brazo Widow-X real.

\subsubsection{Plugins Gazebo}

Para añadir las funcionalidades necesarias para la simulación realista de los modelos en Gazebo ha sido necesario incluir los siguientes plugins:

- Ros_Control: necesario para simular cualquier robot, permite que los controladores puedan actuar sobre las articulaciones.

- Mimic: necesario para las articulaciones de la pinza, donde las dos partes se mueven de manera simétrica y al mismo tiempo.

\subsection{PLATAFORMA MÓVIL TURTLEBOT2}

La plataforma Turtlebot2 está basada en una base diferencial Kobuki [11] que proporciona energía para el ordenador externo, sensores (cámara kinect, encoders magnéticos incrementales, IMU, bumpers, sensores anticaída y antivuelco), actuadores, indicadores y botones. El modelo de la plataforma ya estaba disponible en el repositorio $R O S$, junto con el stack de software de navegación que se utiliza en el experimento 2. Sin embargo, para que su aspecto visual sea más real se ha modificado la geometría de colisión para que coincida con la de CRUMB. 


\subsection{MANIPULADOR MÓVIL $C R U M B$}

Uniendo los modelos del brazo Widow- $X$ y de la plataforma Turtlebot2 se ha obtenido el robot completo $C R U M B$, mostrado en la figura 5 .

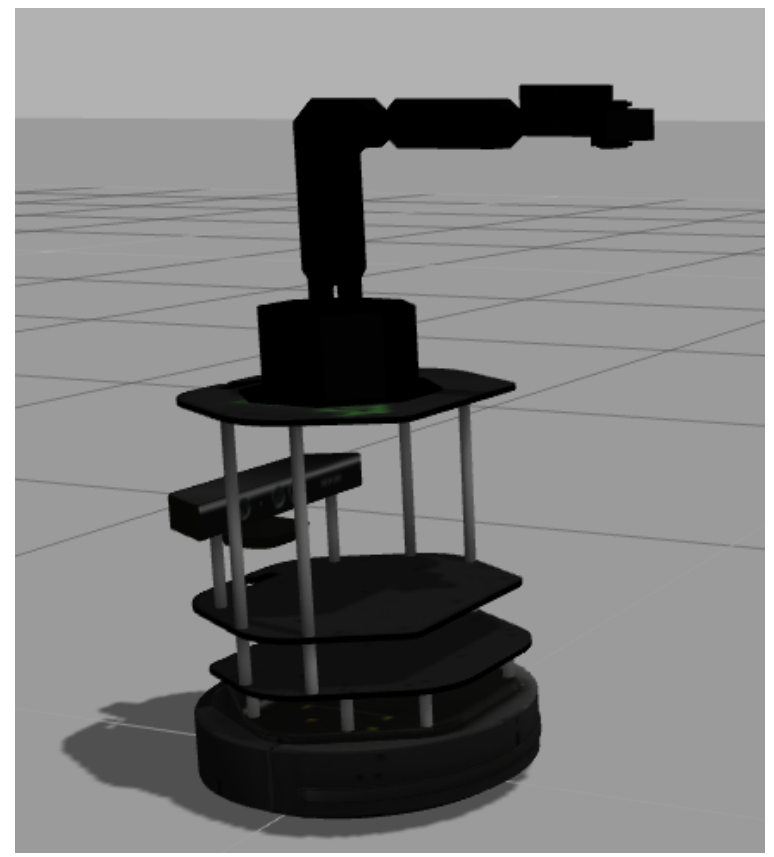

Figura 5: Modelo completo de CRUMB en Gazebo.

Este modelo viene ya asociado en $R O S$ con los topics de programación más importantes existentes en el robot real:

- Comandos de actuación y estado de todas las articulaciones del brazo

- Lectura de los sensores $I M U$, cámara kinect, bumpers, sensores anticaída.

- Datos de odometría de la plataforma.

- Datos del estado completo del sistema, base móvil y brazo.

Sin embargo, otros topics del robot real han tenido que ser incluidos adicionalmente:

- Lectura de los sensores wheel drop y LEDs indicadores.

- Lectura de todos los sensores de la plataforma en un mismo topic.

También se han añadido topics útiles para simulación que no existen en real, como la postura real de la plataforma, el tiempo real y el de simulación.

Finalmente, para que el modelo sea más realista, se ha implementado ruido simulado en el sensor inercial y en la cámara kinect, obteniéndose así 2 modelos de CRUMB simulado distintos, uno con ruido y otro sin ruido, una característica que no es habitual en la simulación con Gazebo y que puede ser muy útil para realizar experimentos de diversos tipos.

Como ejemplo de esta última característica, la figura 6 muestra la lectura de la cámara kinect $\sin$ ruido y con ruido gaussiano.
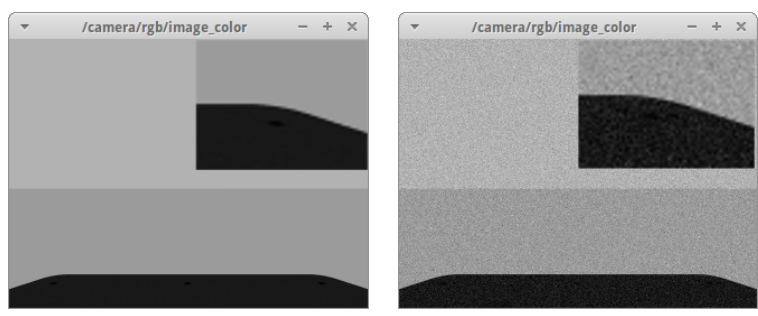

Figura 6: Cámara kinect sin ruido (izda) y con ruido (dcha).

\section{EXPERIMENTOS}

Para validar el trabajo presentado aquí y mostrar su utilidad, se han desarrollado tres aplicaciones experimentales, que se detallan a continuación.

\subsection{EXPERIMENTO 1. PICK-AND-PLACE}

En este experimento el robot permanece fijo en el escenario mostrado en la figura 7 mientras el brazo va moviendo los objetos según programa el usuario. Hay tres posiciones posibles de pick-andplace: dos para los objetos y una libre.

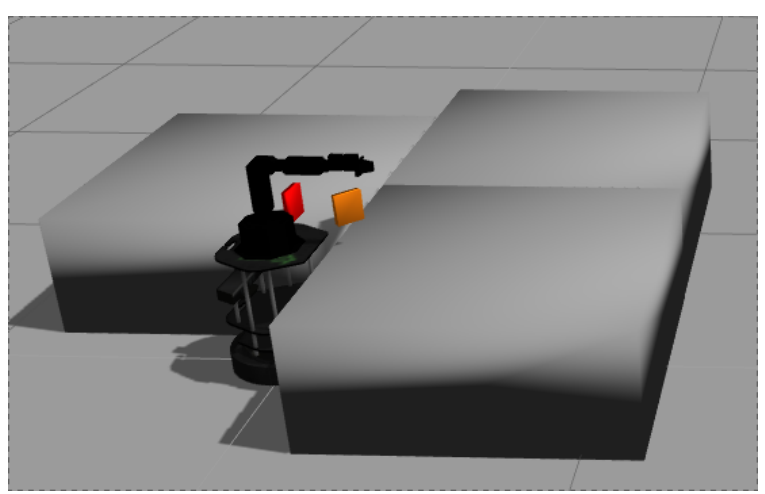

Figura 7: Escenario para pick-and-place.

En la figura 8 se observa cómo $C R U M B$ va moviendo los objetos hacia el hueco libre. En la referencia [12] se encuentra el vídeo para ver la secuencia completa. 

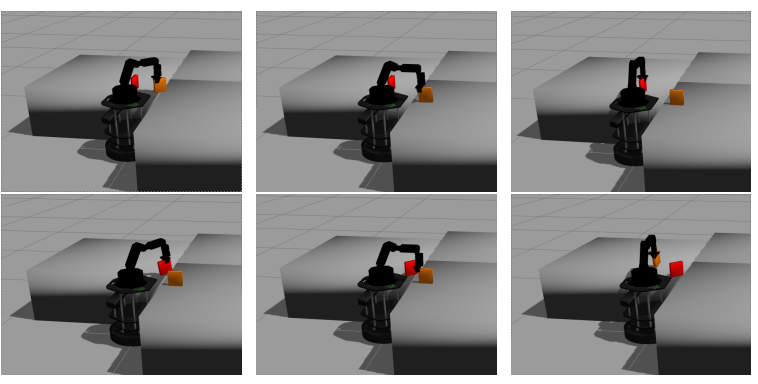

Figura 8: Secuencia de movimiento pick-and-place.

\subsection{EXPERIMENTO 2. MAPEADO DEL ENTORNO Y NAVEGACIÓN}

En el segundo experimento, con el stack de navegación de $R O S, C R U M B$ crea un mapa de su entorno para, posteriormente, navegar a una posición indicada evitando obstáculos. En la figura 9 se muestra el escenario creado en Gazebo.
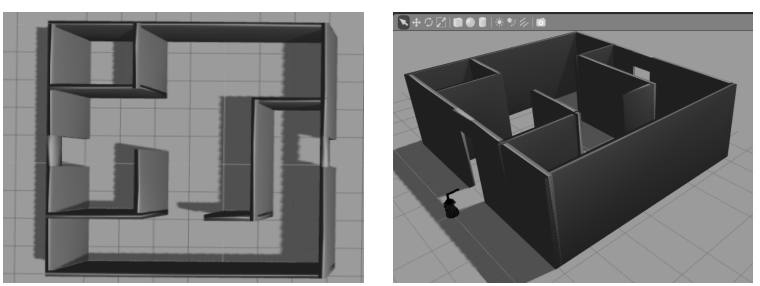

Figura 9: Escenario para mapeado y navegación.

Una vez lanzados los nodos del stack de navegación de $R O S$, se genera el mapa de la figura 10 con los datos recopilados por la cámara kinect.

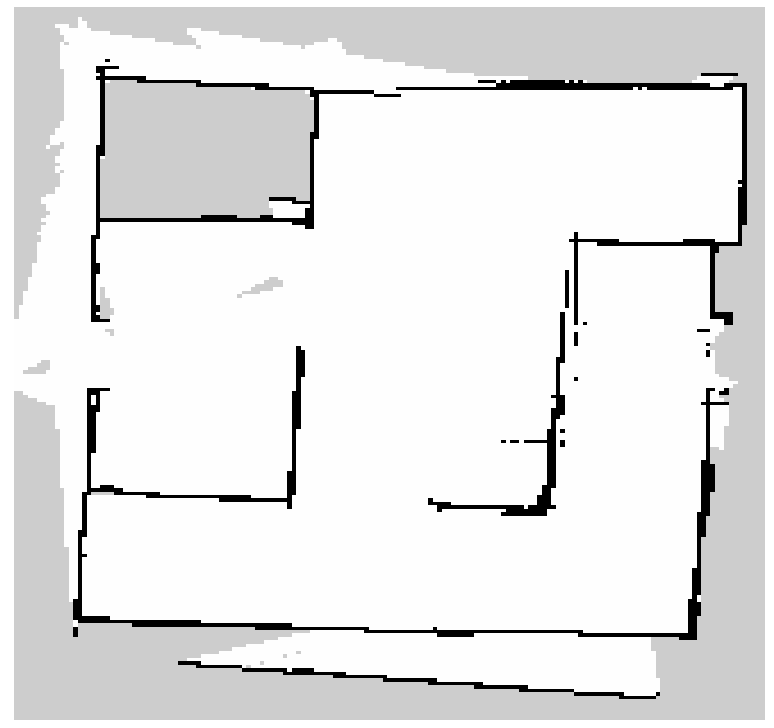

Figura 10: Mapa creado del entorno.

En la figura 11 se observa cómo $C R U M B$ es capaz de ir a un punto indicado en el mapa esquivando las paredes del entorno. Lo más relevante respecto al presente trabajo es que dicha navegación se realiza de manera físicamente realista.
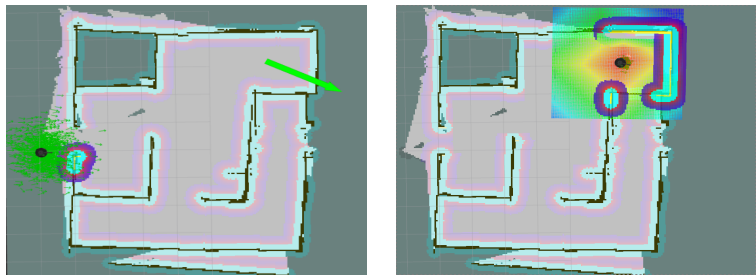

Figura 11: CRUMB llegando al punto objetivo.

Si se incluyeran nuevos objetos en el escenario, utilizando el mismo mapa, el robot llega al punto objetivo esquivando con éxito estos nuevos objetos, como se aprecia en la figura 12.
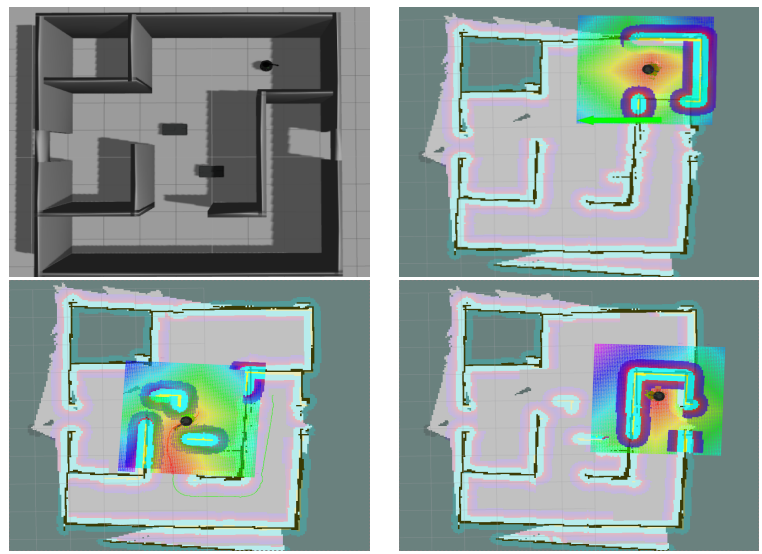

Figura 12: $C R U M B$ navega en un escenario con obstáculos.

En la referencia [13] se puede ver la secuencia completa de este experimento.

\subsection{EXPERIMENTO 3. MANIPULACIÓN MÓVIL}

En este tercer experimento se combina la navegación con la manipulación de objetos. En el escenario de la figura $13 C R U M B$ navega hasta una superficie donde se encuentra un objeto, lo recoge y lo lleva hasta otra superficie para soltarlo.

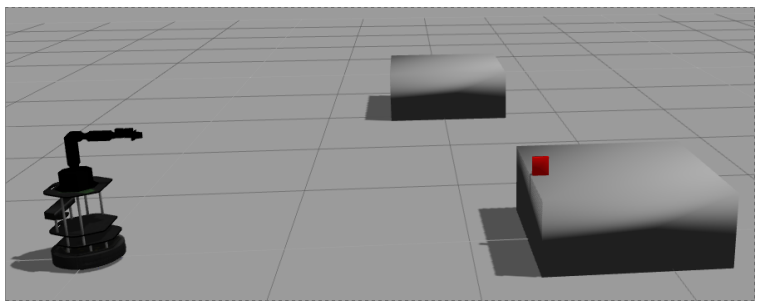

Figura 13: Escenario para la manipulación móvil.

En la figura 14 se ve cómo $C R U M B$ es capaz de realizar la tarea. En la referencia [14] se encuentra 
el vídeo con la secuencia completa de este experimento, donde se puede apreciar mejor el realismo de la simulación.
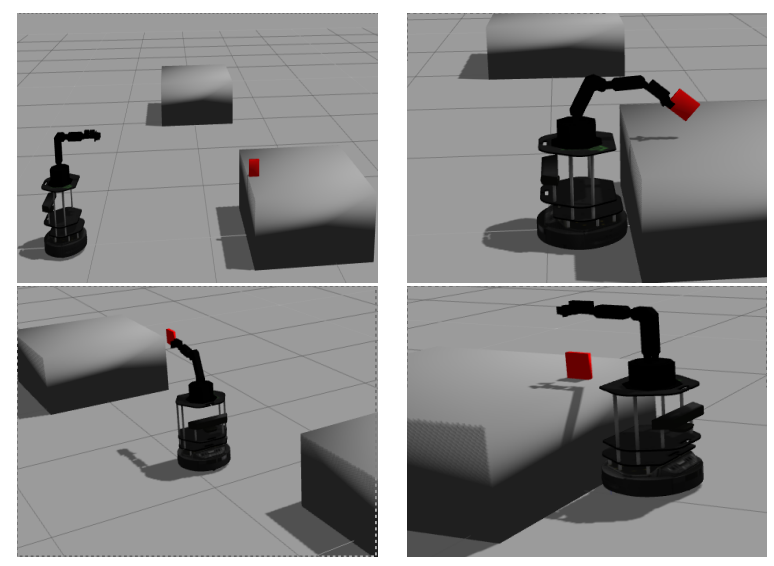

Figura 14: $C R U M B$ realizando el experimento 3.

\section{CONCLUSIONES Y LÍNEAS DE TRABAJO FUTURAS}

En este trabajo se ha presentado una simulación en $R O S$ y Gazebo del manipulador móvil de bajo coste $C R U M B$, compuesto por un brazo Widow-X y una plataforma Turtlebot2. Esta simulación permite ejecutar las mismas aplicaciones que se ejecutarían en el robot real programándolas de modo prácticamente idéntico gracias a que se han añadido todos los topics de ROS necesarios. Además, al incluir elementos geométricos (visuales y de colisión), parámetros físicos, de control y ruido en los sensores $I M U$ y en la cámara kinect, se ha conseguido que el modelo tenga un aspecto muy realista.

Este trabajo también ha incluido el modelado cinemático del brazo Widow-X, no disponible anteriormente, así como la resolución de la cinemática directa e inversa del mismo.

Para validar la simulación se han llevado a cabo diversos experimentos con éxito: pick-and-place, creación de mapas, navegación con obstáculos y navegación combinada con manipulación.

También se han escrito tres manuales: de instalación, uso y desarrollo, y se ha subido todo el código necesario para su uso al popular repositorio de software GitHub del proyecto [15], donde está disponible públicamente.

El trabajo futuro incluye de manera natural el uso de esta simulación en docencia en ingeniería, donde se debería evaluar el aprendizaje del alumno y las diferencias encontradas con el robot real; asimismo, este trabajo ha constituido una base importante del proyecto $C R U M B$, donde se pretende en el futuro implementar diversas líneas de investigación relacionadas con la Robótica Cognitiva en la que los integrantes del proyecto trabajan actualmente, como el aprendizaje por refuerzo o la inferencia bayesiana aplicada a sistemas de percepción robóticos.

\section{Agradecimientos}

Este trabajo ha sido financiado por el Plan Propio de Investigación de la Universidad de Málaga. Los autores agradecen a la empresa Robotnik Automation S.L.L su amabilidad y disposición, así como los ficheros CAD del brazo. También desean agradecer a los compañeros integrantes del proyecto $C R U M B$ sus valiosas aportaciones durante la realización de este trabajo.

\section{English summary}

\section{REALISTIC SIMULATION OF THE LOW-COST TURTLEBOT2 $+W I D O W-X$ MOBILE MANIPU- LATOR IN ROS}

\begin{abstract}
The combination of a Turtlebot2 mobileplatform with a Widow-X robot-arm offers an interesting low-cost solution for research, teaching, or generic educational purposes. Unfortunately, neither does this hardware combination have a complete simulator in the robot programmingframework ROS, nor does the manufacturer offer a kinematic model of the robotic arm. In this article we propose a solution for both issues related to our mobile manipulator. Besides, we describe how robot simulation was developed in Gazebo, a realistic simulator integrated with $R O S$, and the possibility to include simple sensor-noise, which is not always available on most simulators. Finally, we demonstrate the applicability of our simulator by means three experiments that involve pickand-place, mapping, and navigation tasks.
\end{abstract}

Keywords: mobile manipulator, robot simulation, kinematic modelling, Turtlebot2, Widow-X, ROS, Gazebo. 


\section{Referencias}

[1] Blog CRUMB. [Online]. Disponible: http://babel.isa.uma.es/crumb/, Accedido: $20 / 06 / 2018$.

[2] Turtlebot. [Online]. Disponible: http://www.turtlebot.com, Accedido: $20 / 06 / 2018$.

[3] Widow-X. [Online]. Disponible: http://www.trossenrobotics.com/widowxrob otarm, Accedido: 20/06/2018.

[4] Robot Operating System (ROS). [Online]. Disponible: http://www.ros.org, Accedido: 20/06/2018.

[5] Gazebo. [Online]. Disponible: gazebo sim.org/, Accedido: 20/06/2018.

[6] J. J. Craig, Introduction to robotics: mechanics and control, vol. 3. Pearson Prentice Hall Upper Saddle River, 2005.

[7] Plugins Gazebo. [Online]. Disponible: http://gazebosim.org/tutorials?tut=ros_gzpl ugins, Accedido: 09/06/2018.

[8] Robotnik Automation S.L.L. [Online]. Disponible: http://www.robotnik.es/, Accedido: 20/06/2018.

[9] MeshLab [Online]. Disponible: http://www.meshlab.net/ , Accedido: 20/06/2018.

[10] V-REP. [Online]. Disponible: http://www.coppeliarobotics.com/, Accedido: 20/06/2018.

[11] Sensores Kobuki [Online]. Disponible: http://www.robotnik.es/web/wp-content /uploads/2014/04/TB_robot.pdf , Accedido: 20/06/2018.

[12] Vídeo aplicación 1. [Online]. Disponible: http://babel.isa.uma.es/crumb/index.php/2 016/12/04/crumb-grasping-objects/, Accedido: $20 / 06 / 2018$.

[13] Vídeo aplicación 2. [Online]. Disponible: http://babel.isa.uma.es/crumb/index.php/2 016/12/04/navigation-with-crumb/, Accedido: $20 / 06 / 2018$.

[14] Vídeo aplicación 3. [Online]. Disponible: http://babel.isa.uma.es/crumb/index.php/2 016/12/04/crumb-moving-objects-around/, Accedido: 20/06/2018.

[15] Repositorio GitHub [Online]. Disponible: https://github.com/, Accedido: 20/06/2018.

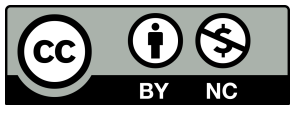

(C) 2018 by the authors. Submitted for possible open access publication under the terms and conditions of the Creative Commons Attribution CC-BY-NC 3.0 license (http://creativecommons.org/licenses/by-nc/3.0/). 